\title{
Ueber einen algebraischen Typus der Bedingungen eines bewegten Massensystems.
}

\author{
(Von Herrn R. Lipschitz in Bonn.)
}

1.

Wenn ein System materieller Punkte unter der Einwirkung eines Systems von Kräften steht, bei dem eine Kräftefunction existirt, und einem System von Bedingungsgleichungen unterworfen ist, die von der Zeit nicht abhängen, so giebt es bekanntlich immer ein Integral, in dessen Ausdruck die individuelle Beschaffenheit der Bedingungen nicht eingeht, das Integral der lebendigen Kraft. Es ist aber, soviel ich weiss, bisher nicht bemerkt worden, dass aus den Gesetzen der Bewegung ein zweites von der individuellen Beschaffenheit der Bedingungen unabhängiges Resultat abgeleitet werden kann, sobald denselben eine gewisse Beschränkung allgemeiner Art auferlegt wird. Nennt man die Punkte des Systems der Reihe nach $\boldsymbol{P}_{1}, \boldsymbol{P}_{2}, \ldots \boldsymbol{P}_{n}$, ferner diejenige Lage desselben, bei der beziehungsweise der Punkt $\boldsymbol{P}_{\alpha}$ den Ort $B_{\alpha}$ einnimmt, $\left(B_{1}, B_{2}, \ldots B_{n}\right)$, und eine gewisse ausgezeichnete Lage $\left(A_{1}, A_{2}, \ldots A_{n}\right)$, bezeichnet man die Coordinaten der Oerter $B_{\alpha}$ und $A_{\alpha}$ in einem rechtwinkligen Coordinatensystem respective durch $x_{\alpha}, y_{\alpha}, z_{\alpha}$ und $a_{\alpha}$, $b_{\alpha}, c_{\alpha}$, endlich die herrschenden Bedingungen durch die Gleichungen

$$
\text { (1.) } \Phi_{1}=0, \quad \Phi_{2}=0, \ldots \Phi_{l}=0,
$$

so lässt sich die in Rede stehende Beschränkung so aussprechen, dass $\Phi_{1}$, $\Phi_{2}, \ldots \Phi_{l}$ algebraische homogene Functionen der $3 n$ Coordinatendifferenzen

$$
x_{\alpha}-a_{\alpha}, y_{\alpha}-b_{\alpha}, \quad z_{\alpha}-c_{\alpha}
$$

sein sollen. Es bedeute nun $\left(B_{1}, B_{2}, \ldots B_{n}\right)$ die Lage, welche das Massensystem während seiner Bewegung zur Zeit $t$ einnimmt, und es werde für jeden Punkt $\boldsymbol{P}_{\alpha}$ das Product aus der demselben zugehörigen Masse $m_{\alpha}$ in das Quadrat des Abstandes $A_{\alpha} B_{\alpha}$ gebildet, und von diesem Product über die $n$ Punkte die Summe genommen, die gleich $2 G$ sein möge, alsdann gilt der Satz, dass der nach der Zeit $t$ genommene zweite Differentialquotient der Summe $2 G$ einen Werth hat, der nicht von der individuellen Beschaffenheit der Bedingungen, sondern allein von der Lage des bewegten Massensystems abhängt. Ich werde diesen Satz beweisen, und mit Hülfe desselben die Bedingungen für die Stabilität der Bewegung bei gewissen Bewegungsproblemen aufstellen. 
In Betreff der allgemeinen Beschaffenheit einer algebraischen homogenen Function $W$ von gegebenen Elementen kann man erwähnen, dass, weil der Grad derselben gleich einem Bruch $\frac{\mathfrak{p}}{\mathfrak{q}}$ ist, dessen Zähler und Nenner ganze Zahlen sind, die Potenz $W^{\mathfrak{a}}$ eine homogene Function vom $\mathfrak{p}^{\text {ten }}$ Grade wird, und deshalb immer einer algebraischen Gleichung

$$
E W^{\text {१s }}+E_{1} W^{\text {q(s-1) }}+\cdots+E_{s}=0
$$

genügen muss, deren Coefficienten $E, E_{1}, \ldots E_{s}$ rationelle ganze homogene Functionen der Elemente von den Graden $e, e+p, \ldots e+p s$ sind. Unter den qs Wurzeln der Gleichung in $W$ werden dann und nur dann zwei Wurzeln von endlichem Werthe gleich, wenn die Discriminante $\Delta$ der Gleichung verschwindet. Schreibt man deshalb den Elementen solche stetige Aenderungen vor, bei denen die Function $A$ nicht durch den Werth Null hindurchgeht, so lassen sich die entsprechenden stetigen Aenderungen einer jeden Wurzel unzweideutig verfolgen. Es werden daher diejenigen Gleichungen, deren Discriminante $\Delta$ die Eigenschaft hat, für kein reelles System der Elemente das Vorzeichen zu wechseln und nur mit allen Elementen zugleich zu verchwinden, unter gewissen Voraussetzungen den Vorzug haben, dass jede reelle Wurzel derselben als eine für alle reellen Werthcombinationen der Elemente reelle, stetige und eindeutige Function der Elemente aufgefasst werden kann; und nur von algebraischen Functionen dieser Beschaffenheit ist im Folgenden stillschweigend die Rede.

Die geometrische Bedeutung des angegebenen Typus der Bedingungsgleichungen ist leicht zu erkennen. Jede Function $\Phi_{\beta}$, wo der Zeiger $\beta$ von 1 bis $l$ lauft, hat nach der Annahme die Eigenschaft, sobald statt der Variabeln $x_{\alpha}, y_{\alpha}, z_{\alpha}$ beziehungsweise die Ausdrücke $a_{\alpha}+p\left(x_{\alpha}-a_{\alpha}\right), b_{\alpha}+p\left(y_{\alpha}-b_{\alpha}\right)$, $c_{\alpha}+p\left(z_{\alpha}-c_{\alpha}\right)$ substituirt werden, wenn der Grad von $\Phi_{\beta}$ gleich $\mathfrak{g}_{\beta}$ ist, für jedes $p$ in den Ausdruck $p^{\mathfrak{g}_{\beta} \boldsymbol{\Phi}_{\beta}}$ überzugehen. Sobald daher für eine Lage $\left(B_{1}, B_{2}, \ldots B_{n}\right)$ die $l$ Gleichungen $\Phi_{\beta}=0$ erfüllt sind, so werden sie auch für jede Lage $\left(B_{1}^{\prime}, B_{2}^{\prime}, \ldots B_{n}^{\prime}\right)$ befriedigt sein, welche zu der ersteren in der Beziehung steht, dass die drei Oerter $\boldsymbol{A}_{\alpha}, \boldsymbol{B}_{\alpha}, \boldsymbol{B}_{\alpha}^{\prime}$ eines jeden Punktes $\boldsymbol{P}_{\alpha}$ auf je einer geraden Linie liegen, und dass der Ort $B_{\alpha}^{\prime}$ die Strecke $A_{\alpha} B_{\alpha}$ nach einem für alle $n$ geraden Linien gleichen Verhältnisse theilt.

Um die ausgesprochene Eigenschaft der Function $G$ zu beweisen, werde die Kräftefunction $U$ genannt, und mögen die Differentialgleichungen für die 
Bewegung des Massensystems nach der Weise von Lagrange mit Anwendung von $l \mathrm{zu}$ bestimmenden Multiplicatoren $\lambda_{1}, \lambda_{2}, \ldots \lambda_{l}$ folgendermassen geschrieben werden:

$$
\left\{\begin{array}{l}
m_{a} \frac{d^{2} x_{\alpha}}{d t^{2}}=\frac{\partial U}{\partial x_{\alpha}}+\lambda_{1} \frac{\partial \Phi_{1}}{\partial x_{\alpha}}+\cdots+\lambda_{l} \frac{\partial \Phi_{l}}{\partial x_{\alpha}} \\
m_{\alpha} \frac{d^{2} y_{\alpha}}{d t^{2}}=\frac{\partial U}{\partial y_{\alpha}}+\lambda_{1} \frac{\partial \Phi_{1}}{\partial y_{\alpha}}+\cdots+\lambda_{l} \frac{\partial \Phi_{l}}{\partial y_{\alpha}} \\
m_{\alpha} \frac{d^{2} z_{\alpha}}{d t^{2}}=\frac{\partial U}{\partial z_{\alpha}}+\lambda_{1} \frac{\partial \Phi_{1}}{\partial z_{\alpha}}+\cdots+\lambda_{l} \frac{\partial \Phi_{l}}{\partial z_{\alpha}}
\end{array}\right.
$$

Ich multiplicire diese drei Gleichungen der Reihe nach mit den Factoren $x_{\alpha}-a_{\alpha}, y_{\alpha}-b_{\alpha}, z_{\alpha}-c_{\alpha}$, addire dieselben und nehme die Summe des Aggregats über die $n$ Punkte des Systems, dann entsteht die Gleichung

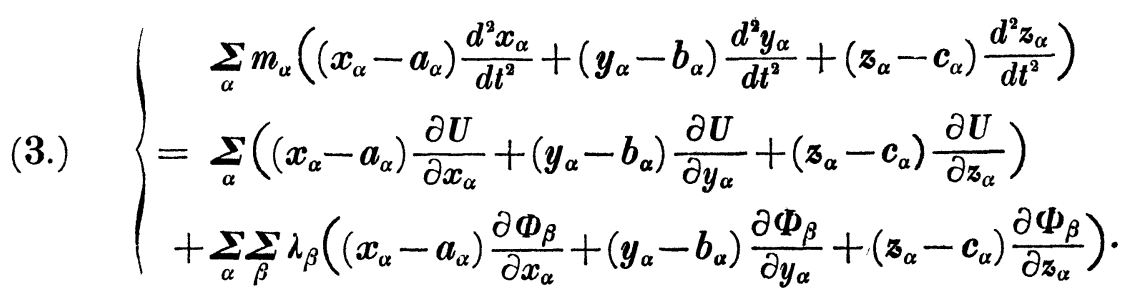

Die Grundeigenschaft der homogenen Functionen liefert aber die Gleichung

$$
\text { (4.) } \quad \boldsymbol{\Sigma}_{\alpha}\left(\left(\boldsymbol{x}_{\alpha}-\boldsymbol{a}_{\alpha}\right) \frac{\partial \Phi_{\beta}}{\partial x_{\alpha}}+\left(\boldsymbol{y}_{\alpha}-\boldsymbol{b}_{\alpha}\right) \frac{\partial \Phi_{\beta}}{\partial y_{\alpha}}+\left(\boldsymbol{z}_{\alpha}-c_{\alpha}\right) \frac{\partial \Phi_{\beta}}{\partial z_{\alpha}}\right)=\mathfrak{g}_{\beta} \boldsymbol{\Phi}_{\beta},
$$

deren rechte Seite wegen der Gleichungen (1.) verschwindet. Demnach erhält die auf der rechten Seite von (3.) befindliche Doppelsumme den Werth Null, und wenn man die linke Seite derselben Gleichung durch Relationen umformt, die den drei Coordinaten entsprechen und nach dem Typus

$$
\frac{d^{2}\left(x_{\alpha}-a_{\alpha}\right)^{2}}{d t^{2}}=2\left(\frac{d x_{\alpha}}{d t}\right)^{2}+2\left(x_{\alpha}-a_{\alpha}\right) \frac{d^{2} x_{\alpha}}{d t^{2}}
$$

gebildet sind, so kommt

$$
\left\{\begin{array}{c}
\frac{1}{2} \sum_{\alpha} m_{\alpha}\left(\frac{d^{2}\left(x_{\alpha}-a_{\alpha}\right)^{2}}{d t^{2}}+\frac{d^{2}\left(y_{\alpha}-b_{\alpha}\right)^{2}}{d t^{2}}+\frac{d^{2}\left(z_{\alpha}-c_{\alpha}\right)^{2}}{d t^{2}}\right)-\sum_{\alpha} m_{\alpha}\left(\left(\frac{d x_{\alpha}}{d t}\right)^{2}+\left(\frac{d y_{\alpha}}{d t}\right)^{2}+\left(\frac{d z_{\alpha}}{d t}\right)^{2}\right) \\
=\sum_{\alpha}\left(\left(x_{\alpha}-a_{\alpha}\right) \frac{\partial U}{\partial x_{\alpha}}+\left(y_{\alpha}-b_{\alpha}\right) \frac{\partial U}{\partial y_{\alpha}}+\left(z_{\alpha}-c_{\alpha}\right) \frac{\partial U}{\partial z_{\alpha}}\right) .
\end{array}\right.
$$

Schon oben ist die Summe, deren allgemeines Glied das Product aus der Masse $m_{\alpha}$ in das Quadrat des Abstandes $A_{\alpha} B_{\alpha}$ ist,

$$
\sum_{\alpha} m_{\alpha}\left(\left(x_{\alpha}-a_{\alpha}\right)^{2}+\left(y_{\alpha}-b_{\alpha}\right)^{2}+\left(z_{\alpha}-c_{\alpha}\right)^{2}\right)=2 G
$$

gesetzt worden, nun wird die Summe der lebendigen Kräfte 


$$
\sum_{\alpha} m_{\alpha}\left(\left(\frac{d x_{\alpha}}{d t}\right)^{2}+\left(\frac{d y_{\alpha}}{d t}\right)^{2}+\left(\frac{d z_{\alpha}}{d t}\right)^{2}\right)=2 T
$$

angenommen, so verwandelt sich die Gleichung (5.) in die folgende

$$
\text { (6.) } \frac{d^{2} \boldsymbol{G}}{d t^{2}}-2 \boldsymbol{T}=\Sigma_{\alpha}\left(\left(\boldsymbol{x}_{\alpha}-\boldsymbol{a}_{\alpha}\right) \frac{\partial U}{\partial x_{\alpha}}+\left(y_{\alpha}-b_{\alpha}\right) \frac{\partial U}{\partial y_{\alpha}}+\left(z_{\alpha}-c_{\alpha}\right) \frac{\partial U}{\partial z_{\alpha}}\right) \text {. }
$$

Hierauf erhält man das Integral der lebendigen Kraft in der folgenden Gestalt

$$
\text { (7.) } \quad T-U=T(0)-U(0),
$$

wo $T(0)$ und $U(0)$ diejenigen Werthe der Functionen $T$ und $U$ bedeuten, welche entstehen, indem man in dieselben für die Ausdrücke $x_{\alpha}, y_{\alpha}, z_{\alpha}$, $\frac{d x_{\alpha}}{d t}, \frac{d y_{\alpha}}{d t}, \frac{d z_{\alpha}}{d t}$ die für einen bestimmten Zeitmoment $t_{0}=t$ gegebenen Werthe der betreffenden Grössen substituirt. Die Elimination der Function $T$ aus den Gleichungen (6.) und (7.) liefert alsdann das Resultat

(8.) $\frac{d^{2} \boldsymbol{G}}{d \boldsymbol{t}^{2}}=\mathbf{2} \boldsymbol{U}+\boldsymbol{\Sigma}_{\alpha}\left(\left(\boldsymbol{x}_{\alpha}-\boldsymbol{a}_{\alpha}\right) \frac{\partial U}{\partial x_{\alpha}}+\left(y_{\alpha}-b_{\alpha}\right) \frac{\partial U}{\partial y_{\alpha}}+\left(\boldsymbol{z}_{\alpha}-c_{\alpha}\right) \frac{\partial \boldsymbol{U}}{\partial z_{\alpha}}\right)+\mathbf{2 T}(\mathbf{0})-\mathbf{2 U}(\mathbf{0})$, durch welches der Behauptung gemäss der zweite auf die Zeit $t$. bezogene Differentialquotient der Grösse $G$ als eine reine Function der Lage des bewegten Massensystems dargestellt wird.

Die Gleichungen (6.) und (8.) nehmen eine noch einfachere Form an, wenn die Kräftefunction $U$ ebenso, wie die Functionen $\Phi_{\beta}$, eine algebraische homogene Function der Elemente $x_{\alpha}-a_{\alpha}, y_{\alpha}-b_{\alpha}, z_{\alpha}-c_{\alpha}$ wird. Bezeichnet man die Ordnung der Function $U$ alsdann mit $\mathfrak{k}$, und schreibt in der Gleichung (4.) $U$ statt $\Phi_{\beta}$ und $\mathfrak{l}^{2}$ statt $\mathfrak{g}_{\beta}$, so führt die hervorgehende Relation zu den neuen Gleichungen

$$
\begin{array}{ll}
\left(6^{*} .\right) & \frac{d G^{2}}{d t^{2}}-2 T=\mathfrak{t} U, \\
\left(8^{*} .\right) & \frac{d^{2} G}{d t^{2}}=(2+\mathfrak{t}) U+2 T(0)-2 U(0) .
\end{array}
$$

Die so eben angestellten Betrachtungen stimmen unter der Voraussetzung, dass die Bewegung des Massensystems frei von Bedingungen ist, in ihrem Wesen mit denjenigen überein, die Jacobi Bd. XVII, pag. 120 dieses Journals und in den Vorlesungen über Dynamik pag. 21 entwickelt hat. Dort fallen aber die Punkte, die gegenwärtig $A_{1}, A_{2}, \ldots A_{n}$ genannt worden sind, in einen zusammen, und dieser ist zum Anfangspunkt der rechtwinkligen Coordinaten genommen. Hierzu kommt bei der von Jacobi gegebenen Anwendung auf ein System von Massen, die allein unter dem Einfluss der gegenseitigen Anziehung stehen, der besondere Umstand, dass die Wahl dieses Coordinaten- 
anfangspunktes für das Problem gleichgültig ist, und deshalb erhält daselbst der nach der Zeit $\boldsymbol{t}$ genommene zweite Differentialquotient der Function $\boldsymbol{G}$ immer den gleichen Werth, in welchen festen Punkt man die Punkte $A_{1}$, $A_{2}, \ldots A_{n}$ auch vereinigen möge.

2.

Wenn man annimmt, dass die sämmtlichen $\Phi_{\beta}$ rationale ganze Functionen ersten Grades von den Elementen $x_{\alpha}-a_{\alpha}, y_{\alpha}-b_{\alpha}, z_{\alpha}-c_{\alpha}$ sind, die Kräftefunction $U$ jedoch eine rationale ganze Function zweiten Grades von diesen Elementen ist, so hat man die allgemeinen Voraussetzungen, welche zutreffen, sobald das Massensystem um die Iage $\left(A_{1}, A_{2}, \ldots A_{n}\right)$ kleine Schwingungen beschreibt*). Aus der Theorie dieses Problems kennt man die Bedingungen, welche nothwendig und himreichend sind, damit für ganz beliebige Anfangsgeschwindigkeiten und Anfangsörter der Massenpunkte weder die Entfernung eines Massenpunktes von der Lage $\left(A_{1}, A_{2}, \ldots A_{n}\right)$ noch die Geschwindigkeit eines Massenpunktes zu irgend einer Zeit gewisse feste Werthe überschreite, und damit das Massensystem in keiner anderen Lage als in der Lage $\left(A_{1}, A_{2}, \ldots A_{n}\right)$ ruhen könne. Es ist aber eine durchgreifende Eigenschaft des charakterisirten algebraischen Typus der Bedingungsgleichungen, dass überall, wo derselbe auftritt, und wo zugleich die Kräftefunction $U$ eine algebraische homogene Function der Elemente $x_{\alpha}-a_{\alpha}, y_{\alpha}-b_{\alpha}, z_{\alpha}-c_{\alpha}$ ist, welche gleich Null wird, sobald alle Elemente zugleich verschwinden, und welche immer in's Unendliche wächst, sobald ein Element jede Grenze überschreitet, die entsprechende, die Stabilität der Bewegung betreffende Frage ebenso einfach beantwortet werden kann, wie bei dem Fall der kleinen Schwingungen. Die zugehörige Antwort lässt sich nämlich, wie folgt, zusammenfassen. Wenn sowohl die sämmtlichen Functionen $\boldsymbol{\Phi}_{\beta}$, wie auch die Kräftefunction $U$ algebraische homogene Functionen der Elemente $x_{\alpha}-a_{\alpha}$, $y_{\alpha}-b_{\alpha}, z_{\alpha}-c_{\alpha}$ sind, wenn die Function $U$ gleich Null wird, sobald alle Elemente in den Werth Null übergehen, und allemal in's Unendliche zunimmt, sobald ein Element über jedes Mass hinaus wächst, so dass der Grad der Function gleich einer positiven Zahl ist, alsdann besteht die nothwendige und hinreichende Bedingung dafür, dass bei ganz beliebigen Anfangsörtern and Anfangsgeschwindigkeiten weder die Entfernung eines Massenpunktes von der Lage $\left(A_{1}, A_{2}, \ldots A_{n}\right)$ noch die Geschwindigkeit eines Massenpunktes zu irgend

*) Lagrange, mécanique analytique, seconde partie, section VI. 
einer Zeit gewisse feste Grenzen überschreite, und dass eine Ruhe des Massensystems ausserhalb der Lage $\left(A_{1}, A_{2}, \ldots A_{n}\right)$ unmöglich sei, darin, dass die Kräftefunction $U$ für alle endlichen, reellen mit den Bedingungen $\Phi_{\beta}=0$ vereinbaren Systeme von Elementen, das System $x_{\alpha}-a_{\alpha}=0, y_{\alpha}-b_{\alpha}=0$, $z_{\alpha}-c_{\alpha}=0$ ausgenommen, einen endlichen negativen Werth erhält. Die Rechtfertigung für dieses Criterium der Stabilität der Bewegung soll sogleich erfolgen.

Zuerst wird gezeigt werden, dass wenn die Bewegung des Massensystems den vorgeschriebenen Charakter der Stabilität bewahrt, die in Rede stehende Function $U$ die angegebene Beschaffenheit haben muss. Zu diesem Behuf eliminire ich aus der nach der Voraussetzung geltenden Gleichung $\left(6^{*}\right.$.) und dem Integral der lebendigen Kraft (7.) die Function $U$ und erhalte die Gleichung

$$
\text { (9.) } \quad(2+\mathfrak{t}) T=\frac{d^{2} G}{d t^{2}}+\mathfrak{l}(T(0)-U(0)) .
$$

Die beiden Seiten derselben, welche in jedem Augenblick der Bewegung vollständig bestimmte Functionen der Zeit $t$ darstellen, können mit dem Element $d t$ multiplicirt und von dem Werthe $t=\sigma$ bis zu dem Werthe $t=\tau$ integrirt werden. Dies giebt das Resultat

$$
\text { (10.) } \quad(2+\mathfrak{t}) \int_{\sigma}^{\tau} T d t=\left(\frac{d G}{d t}\right)_{\sigma}^{\tau}+\mathfrak{t}(T(0)-U(0))(\tau-\sigma),
$$

wo die Function $\frac{d G}{d t}$ die Bedeutung hat

$$
\text { (11.) } \frac{d G}{d t}=\sum_{\alpha} m_{\alpha}\left(\left(x_{\alpha}-a_{\alpha}\right) \frac{d x_{\alpha}}{d t}+\left(y_{\alpha}-b_{\alpha}\right) \frac{d y_{\alpha}}{d t}+\left(z_{\alpha}-c_{\alpha}\right) \frac{d z_{\alpha}}{d t}\right)
$$

und der Ausdruck $\left(\frac{d G}{d t}\right)_{\sigma}^{\tau}$ die Differenz der beiden Werthe von $\frac{d G}{d t}$ bedeutet, die den Substitutionen $t=\sigma$ und $t=\tau$ entsprechen. Wenn nun der Voraussetzung gemäss keine der Grössen $x_{\alpha}-a_{\alpha}, y_{\alpha}-b_{\alpha}, z_{\alpha}-c_{\alpha}, \frac{d x_{\alpha}}{d t}, \frac{d y_{\alpha}}{d t}, \frac{d z_{\alpha}}{d t}$ zu irgend einer Zeit der Bewegung eine feste Grenze übersteigen darf, so gilt dasselbe auch von- dem Ausdruck $\frac{d G}{d t}$, und es ist evident, dass der Bruch $\frac{\left(\frac{d G}{d t}\right)_{\sigma}^{\tau}}{\tau-\sigma}$

wenn die Grösse $\tau$ über jedes Mass hinaus wächst, die Grösse $\sigma$ aber fest bleibt, die Null zur Grenze haben muss. Es werde jetzt die Gleichung (10.) durch den Ausdruck $\mathfrak{f}(\tau-\sigma)$ dividirt, und man lasse den Werth 
$\tau$ über jede Zahl hinaus zunehmen, während der Werth $\sigma$ festgehalten wird, so entsteht für den Grenzfall die Gleichung:

$$
\text { (12.) } \quad \frac{2+\mathfrak{l}}{\mathfrak{f}} \lim \cdot \frac{1}{\tau-\sigma} \int_{\sigma}^{\tau} T d t=T(0)-U(0) \text {. }
$$

Die Summe der lebendigen Kräfte $2 T$ kann vermöge ihrer Natur niemals gleich einer negativen Grösse werden, der Werth derselben ist aber unter den bestehenden Voraussetzungen für jede Zeit zwischen festen Grenzen eingeschlossen, und darf nur in dem einen Falle, dass dauernd $x_{\alpha}-a_{\alpha}=0$, $y_{\alpha}-b_{\alpha}=0, z_{\alpha}-c_{\alpha}=0$ ist, dauernd verschwinden. Deshalb hat der Ausdruck $\lim . \frac{1}{\tau-\sigma} \int_{\sigma .}^{\tau} T d t$, wofern nicht dauernd das System $x_{\alpha}-a_{\alpha}=0, y_{\alpha}-b_{\alpha}=0$, $z_{\alpha}-c_{\alpha}=0$ herrscht, immer einen endlichen positiven Werth, und der Bruch $\frac{2+\mathfrak{f}}{\mathfrak{f}}$ ist in Folge der Hypothese ebenfalls eine positive Grösse. Wollte man nun voraussetzen, dass für ein reelles, den Gleichungen $\Phi_{\beta}=0$ genügendes, von dem System $x_{\alpha}=a_{\alpha}, y_{\alpha}=b_{\alpha}, z_{a}=c_{\alpha}$ verschiedenes System von endlichen Werthen $x_{\alpha}, y_{\alpha}, z_{\alpha}$ die Function $U$ entweder einen positiven oder einen verschwindenden oder einen unendlich grossen Werth annehme, so braucht man nur die betreffenden Werthe $x_{\alpha}, y_{\alpha}, z_{\alpha}{ }^{*}$ zu den Coordinaten der entsprechenden Punkte $P_{\alpha}$ für den Zeitmoment $t=t_{0}$ zu wählen, was nach der Hypothese erlaubt ist, um einen Widerspruch herbeizuführen. Die linke Seite der Gleichung (12.) hat alsdann aus den angegebenen Gründen einen end-' lichen positiven Werth; wenn aber für das in Rede stehende Werthsystem $x_{\alpha}, y_{\alpha}, z_{\alpha}$ der Werth $U(0)$ beziehungsweise negativ, oder verschwindend oder unendlich gross wird, so kann man nach der Hypothese in jedem Falle über die dem Zeitpunkt $t=t_{0}$ entsprechenden Geschwindigkeiten der Massenpunkte und demzufolge über den Werth $T(0)$ so verfügen, dass die rechte Seite der Gleichung (12.) respective negativ oder verschwindend oder unendlich gross wird. Durch den hervortretenden Widerspruch ist somit als nothwendig erwiesen, dass die Function $U$ für alle endlichen reellen, mit den Gleichungen $\Phi_{\beta}=0$ vereinbaren Systeme von Elementen, das System $x_{\alpha}-a_{\alpha}=0$, $y_{\alpha}-b_{\alpha}=0, z_{\alpha}-c_{\alpha}=0$ ausgenommen, einen endlichen negativen Werth erhalte.

Nachdem dieser Punkt erledigt ist, hat es keine Schwierigkeit zu beweisen, dass, wenn die Kräftefunction $U$ die vorgeschriebene Beschaffenheit besitzt, ein Gleichgewicht des Systems ausserhalb der Lage $\left(A_{1}, A_{2}, \ldots A_{n}\right)$ unmöglich ist, und dass die Entfernungen der Massenpunkte von dieser Lage 
sowie die Geschwindigkeiten derselben in feste Grenzen eingeschlossen sind. Sollte erstens ein Gleichgewicht in einer von $\left(A_{1}, A_{2}, \ldots A_{n}\right)$ verschiedenen Lage des Systems bestehen können, so hätte man für jeden Werth der Zeit $T=0$, und deshalb wegen der Gleichung (12.) der Voraussetzung zuwider die Gleichung $U(0)=0$. Die Richtigkeit des zweiten Theiles der Behauptung ist eine Folge aus dem Integral der lebendigen Kraft

$$
\boldsymbol{T}-\boldsymbol{U}=\boldsymbol{T}(\mathbf{0})-\boldsymbol{U}(\mathbf{0}) \text {. }
$$

Nach der bestehenden Voraussetzung darf die Function $U$ für alle endlichen mit den Gleichungen $\boldsymbol{\Phi}_{\beta}=\mathbf{0}$ vereinbaren Werthsysteme $\boldsymbol{x}_{\alpha}, \boldsymbol{y}_{\alpha}, \boldsymbol{z}_{\alpha}$, das System $x_{\alpha}=a_{\alpha}, y_{\alpha}=b_{\alpha}, z_{\alpha}=c_{\alpha}$ ausgeschlossen, nur endliche negative Werthe annehmen, für dieses System aber wird $U=0$; daher ist der Ausdruck $T-U$ ein Aggregat von den beiden Functionen $T$ und $-U$, die für alle zulässigen endlichen Werthsysteme $x_{\alpha}, y_{\alpha}, z_{\alpha}, \frac{d x_{\alpha}}{d t}, \frac{d y_{\alpha}}{d t}, \frac{d z_{\alpha}}{d t}$ endlich bleiben und niemals negativ werden, und das Gleiche gilt mithin von dem speciellen Werthe des Aggregats $\boldsymbol{T}(0)-U(0)$. Nun besitzt die Function $\boldsymbol{T}$ durch ihre Natur die Eigenschaft, wenn eine der Grössen $\frac{d x_{\alpha}}{d t}, \frac{d y_{\alpha}}{d t}, \frac{d z_{\alpha}}{d t}$ über jedes Mass hinaus wächst, mitzuwachsen, und die Function $U$ hat laut der allgemeinen oben getroffenen Voraussetzung die Eigenschaft, wenn eine der Grössen $x_{\alpha}-a_{\alpha}, y_{\alpha}-b_{\alpha}, z_{\alpha}-c_{\alpha}$ über jedes Mass hinaus zunimmt, ebenfalls ohne Ende zuzunehmen. Daher sind die Werthe $x_{\alpha}-a_{\alpha}, y_{\alpha}-b_{\alpha}, z_{\alpha}-c_{\alpha}, \frac{d x_{\alpha}}{d t}, \frac{d y_{\alpha}}{d t}, \frac{d z_{\alpha}}{d t}$ unter den obwaltenden Verhältnissen durch die Gleichung $T-U=T(0)-U(0)$ an gewisse endliche zu keiner Zeit zu überschreitende Grenzen gebunden, und das war behauptet worden. Das aufgestellte Criterium für die Stabilität der Bewegung bei den in Rede stehenden Bewegungsproblemen ist demnach vollständig begründet.

3.

Von dem gewonnenen Standpunkte aus können auch diejenigen Bewegungsprobleme betrachtet werden, bei denen sowohl die Functionen $\Phi_{\beta}$ wie auch die Function $U$ algebraische homogene Functionen der Elemente $x_{\alpha}-a_{\alpha}, y_{\alpha}-b_{\alpha}, z_{\alpha}-c_{\alpha}$ sind, und ausserdem die Function $U$ die Eigenschaften hat, für alle endlichen mit den Bedingungen $\Phi_{\beta}=0$ vereinbaren Systeme von Elementen positiv zu sein, nur zu verschwinden, sobald ein Element in's Unendliche wächst, dann und nur dann unendlich gross zu werden, wenn alle Elemente zugleich verschwinden, sich aber so zu verhalten, dass bei dem $\mathbf{A b}$ - 
nehmen aller Elemente das Product aus der Function $U$ und der Function $G$ immer gegen die Null convergirt. Die Zahl $\mathfrak{k}$, welche den Grad der Function $U$ bezeichnet, liegt dann zwischen den Grenzen 0 und -2 , diese selbst ausgeschlossen. Unter diesen Voraussetzungen lässt sich beweisen, dass bei einem endlichen Werthe der Function $G$ das Massensystem nicht im Gleichgewicht verharren kann, dass, wenn die Functionen $G$ und $T$ zu jeder Zeit der Bewegung kleiner als gewisse endliche Grenzen bleiben, die Constante $T(0)-U(0)$ einen endlichen negativen Werth haben muss $\left.{ }^{*}\right)$, und dass, wenn diese Constante einen endlichen negativen Werth hat, die Function $G$ eine endliche Grenze nicht überschreiten kann.

Die Richtigkeit des ersten und des zweiten Theiles der Behauptung folgt aus dem Umstande, dass, sobald die Functionen $G$ und $T$ nicht wachsen können, die Gleichung (12.) in Kraft tritt. Ist $T$ dauernd gleich Null, so muss, wie auch oben bemerkt; $U(0)$ ebenfalls gleich Null sein, und dies ist nach der Voraussetzung mit einem endlichen Werthe der Function $G$ unverträglich. In dem Falle einer wirklichen Bewegung wird aber die Constante $T(0)-U(0)$ gleich dem Product des endlichen negativen Werthes $\frac{2+\mathfrak{t}}{\mathfrak{t}}$ in den endlichen positiven Werth $\lim \cdot \frac{1}{\tau-\sigma} \int_{\sigma}^{\tau} T d t$. Der dritte Theil der Behauptung wird durch die Gleichung der lebendigen Kraft $T-U=T(0)-U(0)$ erledigt. Denn wenn bei einem negativen Werth der Constante $T(0)-U(0)$ die Function $G$ über jede Grenze hinaus wachsen sollte, so müsste nach der Voraussetzung die Function $U$ sich der Null nähern, also die niemals negative Function $T$ der negativen Constante $T(0)-U(0)$ gleich werden, was unmöglich ist.

Ein Unendlichwerden der Function $T$ kann wegen der Gleichung $\boldsymbol{T}-\boldsymbol{U}=\boldsymbol{T}(\mathbf{0})-\boldsymbol{U}(\mathbf{0})$ nur dann eintreten, wenn die Function $\boldsymbol{U}$ ebenfalls in's Unendliche wächst, und nach der Voraussetzung geschieht dies wieder nur dann, wenn die sämmtlichen Elemente $x_{u}-a_{\alpha}, y_{\alpha}-b_{\alpha}, z_{\alpha}-c_{\alpha}$ sich der Null nähern. Multiplicirt man daher die Gleichung der tebendigen Kraft mit der Function $G$ und beachtet, dass das Product $G U$ nach der Voraussetzung beim Verschwinden der sämmtlichen Elemente immer die Null zur Grenze hat, so erkennt man, dass unter den bestehenden Verhältnissen die Function $T$ nur in dem Falle unendlich gross werden kann, wenn zu derselben Zeit das Massensystem in die Lage $\left(A_{1}, A_{2}, \ldots A_{n}\right)$ übergeht und das Product $G T$ gegen die

*) Vgl. die oben angefübrten Stellen bei Jacobi. 
Null convergirt. Es wird deshalb die Function $\boldsymbol{T}$ niemals in's Unendliche wachsen können, sobald ein Umstand den Vorgang verhindert, dass das Product $\boldsymbol{G T}$ beim Abnehmen der sämmtlichen Elemente $x_{\alpha}-a_{\alpha}, y_{\alpha}-b_{\alpha}, z_{\alpha}-c_{\alpha}$ gegen die Null conkergire.

Man kann nun eine einfache Bedingung für das Eintreten eines solchen Umstandes angeben, wofern die Function $\Phi_{\beta}$ und $U$ noch einer ferneren Beschränkung unterworfen sind. Jede Function $V$, welche die Elemente $x_{\alpha}-a_{\alpha}$, $y_{\alpha}-b_{\alpha}, z_{\alpha}-c_{\alpha}$ nur in den $\frac{n(n+1)}{2}$ homogenen Verbindungen

$$
\left(x_{\alpha}-a_{\alpha}\right)\left(x_{\alpha^{\prime}}-a_{\alpha^{\prime}}\right)+\left(y_{\alpha}-b_{\alpha}\right)\left(y_{a^{\prime}}-b_{\alpha^{\prime}}\right)+\left(z_{\alpha}-c_{\alpha}\right)\left(z_{\alpha^{\prime}}-c_{\alpha^{\prime}}\right)
$$

enthält, wo $\alpha$ und $\alpha^{\prime}$ alle Werthe von 1 bis $n$ durchlaufen, genügt offenbar den drei partiellen Differentialgleichungen

$$
\left\{\begin{array}{l}
\sum_{\alpha}\left(\frac{\partial \boldsymbol{V}}{\partial y_{\alpha}}\left(\boldsymbol{z}_{\alpha}-\boldsymbol{c}_{\alpha}\right)-\frac{\partial \boldsymbol{V}}{\partial \boldsymbol{z}_{\alpha}}\left(\boldsymbol{y}_{\alpha}-\boldsymbol{b}_{\alpha}\right)\right)=\mathbf{0} \\
\boldsymbol{\Sigma}_{\alpha}\left(\frac{\partial \boldsymbol{V}}{\partial \boldsymbol{z}_{\alpha}}\left(\boldsymbol{x}_{\alpha}-\boldsymbol{a}_{\alpha}\right)-\frac{\partial \boldsymbol{V}}{\partial \boldsymbol{x}_{\alpha}}\left(\boldsymbol{z}_{\alpha}-\boldsymbol{c}_{\alpha}\right)\right)=\mathbf{0} \\
\underset{\alpha}{\boldsymbol{\Sigma}}\left(\frac{\partial \boldsymbol{V}}{\partial \boldsymbol{x}_{\alpha}}\left(\boldsymbol{y}_{\alpha}-\boldsymbol{b}_{\alpha}\right)-\frac{\partial \boldsymbol{V}}{\partial y_{\alpha}}\left(\boldsymbol{x}_{\alpha}-\boldsymbol{a}_{\alpha}\right)\right)=\mathbf{0} .
\end{array}\right.
$$

Wenn daher die Functionen $\Phi_{\beta}$ und $U$ die Elemente $x_{\alpha}-a_{\alpha}, y_{\alpha}-b_{\alpha}, z_{\alpha}-c_{\alpha}$ nur in den $\frac{n(n+1)}{2}$ homogenen Verbindungen

$$
\left(x_{\alpha}-a_{\alpha}\right)\left(x_{\alpha^{\prime}}-a_{\alpha^{\prime}}\right)+\left(y_{\alpha}-b_{c}\right)\left(y_{\alpha^{\prime}}-b_{\alpha^{\prime}}\right)+\left(z_{\alpha}-c_{\alpha}\right)\left(z_{\alpha^{\prime}}-c_{\alpha^{\prime}}\right)
$$

enthalten, so genügen dieselben den für $V$ aufgestellten drei partiellen Differentialgleichungen, und man kann aus den Differentialgleichungen der Bewegung (2.) die folgenden Gleichungen herleiten:

$$
\left\{\begin{array}{l}
\sum_{\alpha} m_{\alpha}\left(\frac{d^{2} y_{\alpha}}{d t^{2}}\left(z_{\alpha}-c_{\alpha}\right)-\frac{d^{2} z_{\alpha}}{d t^{2}}\left(y_{\alpha}-b_{\alpha}\right)\right)=0 \\
\sum_{\alpha} m_{\alpha}\left(\frac{d^{2} z_{\alpha}}{d t^{2}}\left(x_{\alpha}-a_{\alpha}\right)-\frac{d^{2} x_{\alpha}}{d t^{2}}\left(z_{\alpha}-c_{\alpha}\right)\right)=0 \\
\sum_{\alpha} m_{\alpha}\left(\frac{d^{2} x_{\alpha}}{d t^{2}}\left(y_{\alpha}-b_{\alpha}\right)-\frac{d^{2} y_{\alpha}}{d t^{2}}\left(x_{\alpha}-a_{\alpha}\right)\right)=0
\end{array}\right.
$$

welche sofort die drei Integrale liefern

$$
\left\{\begin{array}{l}
\sum_{\alpha} m_{\alpha}\left(\frac{d y_{\alpha}}{d t}\left(z_{\alpha}-c_{\alpha}\right)-\frac{d z_{\alpha}}{d t}\left(y_{\alpha}-b_{\alpha}\right)\right)=\mathfrak{A} \\
\sum_{\alpha} m_{\alpha}\left(\frac{d z_{\alpha}}{d t}\left(x_{\alpha}-a_{\alpha}\right)-\frac{d x_{\alpha}}{d t}\left(z_{\alpha}-c_{\alpha}\right)\right)=\mathfrak{B} \\
\sum_{\alpha} m_{\alpha}\left(\frac{d x_{\alpha}}{d t}\left(y_{\alpha}-b_{\alpha}\right)-\frac{d y_{\alpha}}{d t}\left(x_{\alpha}-a_{\alpha}\right)\right)=\mathfrak{E}
\end{array}\right.
$$


Dieselben gehen in die Integrale über, welche unter dem Namen der Flächensätze bekannt sind, sobald die $n$ Punkte $A_{1}, A_{2}, \ldots A_{n}$ in einen Punkt vereinigt werden. Ich werde jetzt den Beweis der Thatsache führen, dass, wenn die Quadratsumme der drei neuen Integrationsconstanten $\mathfrak{A}^{2}+\mathfrak{B}^{2}+\mathfrak{S}^{2}$ nicht gleich Null ist, das Product $G T$ beim Abnehmen der sämmtlichen Elemente $x_{\alpha}-a_{\alpha}, y_{\alpha}-b_{\alpha}, z_{\alpha}-c_{\alpha}$ nicht verschwinden kann, und dass folglich alsdann unter den gegebenen Verhältnissen die Function $T$ zu keiner Zeit in's Unendliche zunimmt.

Der Kürze halber mögen die folgenden Bezeichnungen gebraucht werden

dann kommt

$$
\left\{\begin{array}{l}
\frac{d y_{\alpha}}{d t}\left(z_{\alpha}-c_{\alpha}\right)-\frac{d z_{\alpha}}{d t}\left(y_{\alpha}-b_{\alpha}\right)=p_{\alpha}, \\
\frac{d z_{\alpha}}{d t}\left(x_{\alpha}-a_{\alpha}\right)-\frac{d x_{\alpha}}{d t}\left(z_{\alpha}-c_{\alpha}\right)=q_{\alpha}, \\
\frac{d x_{\alpha}}{d t}\left(y_{\alpha}-b_{\alpha}\right)-\frac{d y_{\alpha}}{d t}\left(x_{\alpha}-a_{\alpha}\right)=r_{\alpha}, \\
\left(x_{\alpha}-a_{\alpha}\right)^{2}+\left(y_{\alpha}-b_{\alpha}\right)^{2}+\left(z_{\alpha}-c_{\alpha}\right)^{2}=s_{\alpha}^{2}, \\
\left(\frac{d x_{\alpha}}{d t}\right)^{2}+\left(\frac{d y_{\alpha}}{d t}\right)^{2}+\left(\frac{d z_{\alpha}}{d t}\right)^{2}=v_{\alpha}^{2}, \\
\left(\sum_{\alpha} m_{\alpha} p_{\alpha}\right)^{2}+\left(\sum_{\alpha} m_{\alpha} q_{\alpha}\right)^{2}+\left(\sum m_{\alpha} r_{\alpha}\right)^{2}=D^{2},
\end{array}\right.
$$

$$
\sum_{\alpha} m_{\alpha} s_{\alpha}^{2}=2 G, \quad \sum_{\alpha} m_{\alpha} v_{\alpha}^{2}=2 T .
$$

Wenn nun bei der einfachen Summation, wie bisher, der Buchstabe $\alpha$ die Werthe von 1 bis $n$ durchläuft, dagegen bei der doppelten Summation die Buchstaben $\alpha$ und $\alpha^{\prime}$ nur die Paare ungleicher Zahlen aus der Reihe von 1 bis $n$ darstellen, so gilt die Gleichung

$$
\left\{\begin{aligned}
4 G T-D^{2}= & \sum_{\alpha} m_{\alpha}^{2}\left(s_{\alpha}^{2} v_{\alpha}^{2}-p_{\alpha}^{2}-q_{\alpha}^{2}-r_{\alpha}^{2}\right) \\
& +\sum_{\alpha} \sum_{\alpha^{\prime}} m_{a} m_{\alpha^{\prime}}\left(s_{\alpha}^{2} v_{\alpha^{\prime}}^{2}+s_{\alpha^{\prime}}^{2} v_{\alpha}^{2}-2 p_{\alpha} p_{\alpha^{\prime}}-2 q_{\alpha} q_{\alpha^{\prime}}-2 r_{\alpha} r_{\alpha^{\prime}}\right) .
\end{aligned}\right.
$$

Da allgemein die Ungleichheit

$$
\left(p_{\alpha} p_{\alpha^{\prime}}+q_{\alpha} q_{\alpha^{\prime}}+r_{\alpha} r_{\alpha^{\prime}}\right)^{2} \leqq\left(p_{\alpha}^{2}+q_{\alpha}^{2}+r_{\alpha}^{2}\right)\left(p_{\alpha^{\prime}}^{2}+q_{\alpha^{\prime}}^{2}+r_{\alpha^{\prime}}^{2}\right)
$$

besteht, und für die Grössen $p_{\alpha}, q_{\alpha}, r_{\alpha}$ in Folge ihres Bildungsgesetzes die bekannte Relation

$$
\text { (18.) } \quad p_{\alpha}^{2}+q_{\alpha}^{2}+r_{\alpha}^{2}=s_{\alpha}^{2} v_{\alpha}^{2}-s_{\alpha}^{2}\left(\frac{d s_{\alpha}}{d t}\right)^{2}
$$

stattfindet, so hat man die Folgerungen 


$$
\begin{gathered}
s_{\alpha}^{2} v_{\alpha}^{2}-p_{\alpha}^{2}-q_{\alpha}^{2}-r_{\alpha}^{2}=s_{\alpha}^{2}\left(\frac{d s_{\alpha}}{d t}\right)^{2} \geqq 0, \\
\left(p_{\alpha} p_{\alpha^{\prime}}+q_{\alpha} q_{\alpha^{\prime}}+r_{\alpha} r_{\alpha^{\prime}}\right)^{2}<s_{\alpha}^{2} v_{\alpha}^{2} s_{\alpha^{\prime}}^{2} v_{\alpha^{\prime}}^{2}, \\
s_{\alpha}^{2} v_{\alpha^{\prime}}^{2}+s_{\alpha^{\prime}}^{2} v_{\alpha}^{2}-2 p_{\alpha} p_{\alpha^{\prime}}-2 q_{\alpha} q_{\alpha^{\prime}}-2 r_{\alpha} r_{\alpha^{\prime}} \geqq 0 .
\end{gathered}
$$

Also ist die Verbindung $4 G T-D^{2}$ gleich einem Aggregat von lauter nicht negativen Grössen. In Folge der drei Integrale (15.) wird $D^{2}$ gleich der Constante $\mathfrak{A}^{2}+\mathfrak{B}^{2}+\mathfrak{C}^{2}$, und es leuchtet ein; dass, wenn dieselbe einen von der Null verschiedenen Werth hat, das Product $G T$ auch beim Abnehmen der sämmtlichen Elemente $x_{\alpha}-a_{\alpha}, y_{\alpha}-b_{\alpha}, z_{\alpha}-c_{\alpha}$ nicht verschwinden kann; dies aber war zu beweisen.

Wenn der Zeit $t=t_{0}$ ein endliches, den Bedingungen $\Phi_{\beta}=0$ genügendes, von dem System $x_{\alpha}=a_{\alpha}, y_{\alpha}=b_{\alpha}, z_{\alpha}=c_{\alpha}$ verschiedenes, sonst aber ganz belirebiges Werthsystem $x_{\alpha}, y_{\alpha}, z_{\alpha}$ entspricht, so ist nach der Voraussetzung der zugehörige Werth $U(0)$ ein endlicher und positiver; es kann deshalb durch die Wahl der Geschwindigkeitscomponenten $\frac{d x_{\alpha}}{d t}, \frac{d y_{\alpha}}{d t}, \frac{d z_{\alpha}}{d t}$ für die Zeit $t=t_{0}$ ebensowohl bewirkt werden, dass die Constante $T(0)-U(0)$ einen negativen und die Constante $\mathfrak{U}^{2}+\mathfrak{B}^{2}+\mathfrak{\complement}^{2}$ einen von der Null verschiedenen Werth erhalte, wie auch, dass die Constante $T(0)-U(0)$ einen positiven Werth bekomme. Hieraus zieht man den Schluss, dass, wenn unter den obwaltenden Verhältnissen die Lage des Massensystems beliebig, doch so gegeben ist, dass die Function $G$ einen endlichen von der Null verschiedenen Werth hat, es lediglich von den zugehörigen Werthen der Geschwindigkeitscomponenten abhängt, ob die Bewegung den Charakter der Stabilität an sich trage oder nicht.

Bonn, den 16. October 1866. 